\title{
The New Instructions to Authors for the Reporting of Steroid Hormone Measurements
}

(C) the Endocrine Society 2014

The Endocrine Society Council recently established a "Sex Steroid Assays Reporting Task Force (SSARTF)" with the following charges: to perform a review of journal policies (our journals and sister journals) related to sex hormone measurement reporting requirements; to recommend to Council through the Publications Core Committee (PCC) a sex hormone measurement reporting policy that ensures our journal policies are not contradictory; and to then make recommendations on how to implement and communicate the new policy to authors, reviewers and readers of our journals. The SSARTF met by video conferencing as well as email and phone communication to develop the new Instructions to Authors.

First, our research revealed that few journals have specific policies relating to the reporting of sex hormones or more generally steroid hormone assay measurements. Although the science of optimal assay measurements for many analytes is always in flux, all felt that the Endocrine Society should lead the field towards optimization of measurement of steroid hormones in research publications to impact the validity of the science and ultimately the care of our patients. As the draft guideline was developed, the SSARTF felt that the charge was overly narrowly restricted in specifying sex hormones and that the instructions could be widely applied to all steroid hormones. Our goal was to provide a set of instructions that were clear and concise.

The new Instructions to Authors on the Reporting of Steroid Hormone Assay Measurements addresses important requirements for minimal analytical validity including standards of accuracy, precision, specificity, sensitivity, reproducibility and stability. We realize that a requirement for traceability of an assay to a certified standard is not yet available for all steroid hormones, but is an important goal. Importantly, the instructions do not mandate a specific type of hormone assay, but clearly state the criteria for a valid assay for clinical and non-clinical studies and give examples of where some widely available steroid hormone assays are not sufficient to meet these criteria.
Concerning implementation, the SSARTF suggests that a checklist for authors and reviewers be used to ensure that these criteria are fulfilled or justification for their omission be provided. Initially, the SSARTF suggests that specific "hormone assay expert reviewers" may be needed, similar to the use of "statistical experts", to ensure optimal review of manuscripts with new or complex steroid hormone methodology. We realize that, due to journal-specific word limits, the details of assay methodology might appear in a supplementary data file.

Finally, we appreciate that these new Instructions to Authors are only the first step in an ongoing process. With their implementation, the feedback from authors and reviewers, as well as changes in the scientific landscape of assay measurement, will drive the evolution of these instructions to remain consistent with the state of the science. In parallel, the Endocrine Society will lead the discussion in our scientific manuscripts and editorials as well as educational programing to engage industry, governmental and other stakeholders to support optimization of all hormone assay measurements to common standards. These steps will ensure the validity of our endocrine science and guide the care of patients with endocrine disorders.

Margaret E. Wierman, MD

Chair, Sex Steroid Assays Reporting Task Force

\section{SSARTF Members}

Richard J. Auchus, MD, PhD

Daniel J. Haisenleder, PhD

Janet E. Hall, MD

David Handelsman, MB BS, FRACP, PhD

Susan Hankinson, $\mathrm{ScD}$

William Rosner, MD

Ravinder J. Singh, $\mathrm{PhD}$

Patrick M. Sluss, PhD

Frank Z. Stanczyk, PhD 